\section{Computing in general practice}

Computing in general practice can scarcely be considered news. More than 20 years ago information was being collected and entered onto what were then called "mainframe" computers, in Britain and elsewhere. The explosion into desktop computers produced a rapid expansion of what (at least in the early days) some people would consider "data" about GP activity as opposed to useful "information" about clinical practice in the primary care setting.

Now that questions of usefulness of computerised information are seen as increasingly important, technical developments into networking have understandably replaced those of data entry and basic analysis. Conceptually we can see value in GPs exchanging and comparing information, in hospitals and GPs exchanging information electronically, and also in health commissions or related groups having aggregated information to use in their assessment of communities and their health care. Of course, commercial considerations have always been important, in addition to more general problems of ownership and confidentiality.

This number of the $\mathcal{F E C H}$ presents side by side two papers addressing the feasibility and utility of networked primary care information systems, one in The Netherlands and one in the north of England. It is instructive to see how the nature of the health care system influences both the aims and the delivery of such systems. But such achievements on a wide scale are difficult, and clearly "research" may not be an adequate driving force to motivate primary care teams to collect and share information in this way.

\section{Research, Alzheimer's disease, and glue ear}

Getting research into practice is the title of our guest editorial this month - it comes from a research area where epidemiology, community health, public health and general practice overlap.

Two papers from a research group in the north of England (one full paper and one short report) look at environmental aspects of Alzheimer's disease. There are also two papers looking at aspects of body mass index and health, as well as an interesting historical paper on the affects of famine on infant mortality.

Again, following a review paper on an unusual epidemic - that of surgery for glue ear - we welcome a brief paper from Lithuania looking at basic aspects of health and epidemiology and demonstrated mortality - avoidable mortality in particular.

\title{
Editorial
}

\section{Getting research into practice}

Research often fails to get into practice. ${ }^{1-3}$ It may even take years before new findings are incorporated into the advice of experts. ${ }^{4}$ Health services research is designed to make health care more effective. If its results are to be put into practice, the research questions must be relevant, and the results must be disseminated effectively. However, there is a longstanding cultural divide between researchers, practitioners, and managers. ${ }^{4}$ This divide may be getting worse, as researchers are increasingly driven by the need to produce high quality peer reviewed publications, and as managers are driven by the need to deliver change in ever shorter timescales. For health services research findings to be implemented, the divide between researchers and managers must be overcome. Defining the agenda for health services research is a collective responsibility, which should not be dictated by one party. Indeed, there will be situations where it is appropriate for researchers and health service staff to work closely together throughout the course of a project. The research then becomes part of the development process - both contributing to the development of the service and to its evaluation.
Even if research is relevant, it won't get put into practice if no-one hears about it. Simple distribution of research findings or guidelines is a poor method of changing clinical behaviour. ${ }^{56}$ Strategies which do result in change include educational meetings (though simple instruction seems to be of very little value), ${ }^{78}$ outreach visits, ${ }^{9}$ promotion by local opinion leaders, ${ }^{10}$ individual feedback combined with audit, ${ }^{11}$ computer based decision support systems, ${ }^{12}$ minisabbaticals and, not least, the provision of financial incentives. ${ }^{13}$ Guidelines are most effective when developed locally or adapted to local needs, when linked to educational interventions, and when linked to feedback, especially patient-specific prompts during the consultation. ${ }^{1415}$ Public education campaigns ${ }^{1617}$ or the use of research information by lay pressure groups ${ }^{18}$ also influence professional behaviour from the consumer side.

There are no "magic bullets" for dissemination, ${ }^{5}$ and effective implementation of research findings is likely to require a range of strategies. ${ }^{319-22}$ There is a major educational task for both managers and professionals. For managers, active steps are needed to involve them in 
both commissioning and using research. For doctors, there needs to be a fundamental change in medical education, in line with the General Medical Council's recent recommendations that critical evaluation of evidence should be promoted along with an approach to medicine that is both questioning and self critical. ${ }^{23}$

Who is responsible for disseminating research findings? Both researchers and funding organisations have in the past regarded their job as finished when results are published in peer reviewed journals. Can this be acceptable if it means that research never gets into practice? If not, dissemination will need specific academic recognition and funding. There is also a growing need for systematic reviews of research. Reviews are essential to cope with the increasing volume of research (not all of which is good) and to avoid the dissemination of conflicting research findings. ${ }^{24}$ Governments have a major responsibility in this area. This has been recognised in Britain with the establishment of the Cochrane Centre, ${ }^{25}$ the Centre for Reviews and Dissemination, and a regional network for research and development, and in the United States through the dissemination work of the Agency for Health Care Policy and Research. ${ }^{26}$

A number of health authorities have taken a lead in disseminating the results of research, for example the GRiP initiative (Getting Research into Practice) of the Anglia and Oxford Region. ${ }^{27}$ The purchaser-provider split in the UK NHS offers potential for contracts to be used to influence clinical practice, and there is some evidence that quality statements about clinical effectiveness are beginning to appear in NHS contracts. ${ }^{28}$ While moves towards formal contracts for care may lead to improved outcome, they should not be allowed to detract from other aspects of care, such as forging a close doctor-patient relationship, which are less readily researched, but form an essential part of good medicine. ${ }^{29}$

The NHS in the UK has recently committed itself to producing knowledge-based change by underpinning development with research. ${ }^{30}$ This presents particular challenges for primary care at a time when there is both a major shift of care to the primary sector, and an increase in the importance of general practitioners as purchasers and commissioners of health care. There are, however, real dangers of fragmented purchasing policies from multiple small purchasers. This will increase the difficulty of getting the results of research into contracts or clinical care. The research culture in primary care has traditionally been weak, and the urgent need to address this has been acknowledged in the United Kingdom by the Culyer Report. ${ }^{31}$

Putting research into practice requires a shared understanding between policy makers, managers, purchasers and providers of health care. They all need to be involved in setting the research agenda and in disseminating and implementing the result of research. As Archie Cochrane put it more than 20 years ago: "There is a whole rational health service to gain". 32

MARTIN ROLAND
Director of Research
National Primary Care Research and Development Centre
Universities of Manchester, York and Salford
Manchester M14 5NP

1 Williamson JW, German PS, Weiss R, et al. Health science information management and continuing education of physicians: a survey of US
primary care practitioners and their opinion leaders. Ann Intern Med 1989; 110:151-60.

2 Sheldon TA, Borowitz M. Changing the measure of quality in the NHS: from purchasing activity to purchasing protocols. Quality in Health Care 1993;2:149-50.

3 Haines A, Jones R. Implementing findings of research. BMF 1994;308: 1488-92.

4 Antmann EM, Lau J, Kupelnick B, et al. A comparison of results of metaanalyses of randomised controlled trials and recommendations of clinical analyses of randomised controlled
experts. $\mathcal{F} A M A 1992 ; 268: 240-8$.

5 Lomas J, Haynes BR. A taxonomy and critical review of tested strategies for the application of clinical practice recommendations: from official to for the application of clinical practice recommendations:
individual clinical policy. $A m \mathcal{F}$ Prev Med 1987;4:77-97.

6 Lomas J, Anderson GM, Dominick-Pierre K, et al. Do guidelines guide practice? Effect of a consensus statement on the practice of physicians. practice? Effect of a consensus st.

7 Davis DA, Thomson MA, Oxman AD, Haynes RB. Evidence for the effectiveness of CME: a review of 50 randomised controlled trials. $\mathscr{f} A M A$ 1992;268:1111-7.

8 Waddell D. The effects of continuing education on nursing practice: a metaanalysis. Fournal of Continuing Education in Nursing 1991;22:113-8.

9 Soumerai and Avorn. Principles of educational outreach ("academic detailing”) to improve clinical decision making. $\mathcal{F} A M A$ 1990;253:549-56.

10 Lomas J, Enkin M, Anderson G, et al. Opinion leaders vs audit and feedback to implement practice guidelines. $\mathcal{F} A M A 1991 ; 303: 398-402$.

11 Mugford M, Banfield P, O'Hanlon M. Effects of feedback of information on clinical practice: a review. $B M \mathcal{F}$ 1991;303:398-402.

12 Johnston MF, Langton KB, Haynes B, Mathieu A. Effects of computer based clinical decision support systems on clinician performance and patient outcome: a critical appraisal of research. Ann Intern Med 1994; 120:135-42.

13 Roland MO. Pay more, get more? f Epidemiol Community Health 1993;47: 431.

14 Grimshaw J, Russell IT. Effect of clinical guidelines on medical practice: a systematic review of rigorous evaluations. Lancet 1993;342:1317-22.

15 Grimshaw JM, Russell IT. Achieving health gain through clinical guidelines. 2: Ensuring guidelines change medical practice. Quality in Health Care 1994;3:45-52.

16 Domenighetti G, Lurqaschi P, Gutzwiller, F, et al. Effect of information campaign by the mass media on hysterectomy rates. Lancet 1988;ii:

17 Soumerai S, Ross-Degnan D, Kahn J. Effects of professional and media warnings about the association between aspirin and Reye's syndrome. Millbank $Q$. 1992;70:155-82.

18 Stocking B. Implementing the findings of "Effective care in pregnancy and childbirth" in the UK. Millbank $Q .1993 ; 71: 497-521$.

19 Atkinson C, Hayden J. Managing change in primary care: strategies for success. BMF 1992;304:1488-90.

20 Horder J, Bosanquet N, Stocking B. Ways of influencing the behaviour of general practitioners. $\mathcal{F} R$ Coll Gen Pr 1986;36:517-21.

$21 \mathrm{Grol}$ R. Implementing guidelines in general practice care. Quality in Health Care 1992;1:184-91.

22 Stocking B. Promoting change in clinical care. Quality in Health Care 1992 1:56-60.

23 General Medical Council. Tomorrow's doctors: recommendations on un dergraduate education. 1993. London: GMC.

24 Angell M, Kassiror JP. Clinical research: what should the public believe? $N$ Eng $\mathcal{F}$ Med 1994;331:189-90.

25 Chalmers I, Dickersin K, Chalmers TC. Getting to grips with Archie Cochrane's agenda. BMf 1992;305:786-8.

26 Goldberg HI, Cummings MA, Steinberg EP, et al. Deliberations on the dissemination of PORT products: translating research findings into imdissemination of PORT products: translating research 10

27 Dunning M, McQuay H, Milne R. Getting a GRiP. Health Service fournal 1994;104:24-6.

28 Lord J, Littlejohns P. Secret garden. Health Service fournal 1994;104:18-20.

28 Lord J, Littlejohns P. Secret garden. Health Service fournal 1994;104:18-20. Royal College of General Practitioners. 1994.

Royal College of General Practitioners. 1994.
Department of Health. Research for health 1993. London, HMSO.

30 Department of Health. Research for health 1993 . London, HMSO.

Department of Health. Supporting research and development in the NHS.
Report of the Research and Development Task Force. London: HMSO, 1994.

32 Cochrane A. Effectiveness and efficiency. London: HMSO, Nuffield Provincial Hospitals Trust, 1972. 